\title{
Relapsed childhood acute lymphoblastic leukemia: Experience from a single tertiary center in Thailand
}

\author{
Thirachit Chotsampancharoen ${ }^{1}$, Natsaruth Songthawee ${ }^{1}$, Shevachut Chavananon ${ }^{1}$, \\ Pornpun Sripornsawan ${ }^{1}$, and Edward McNeil ${ }^{2}$ \\ ${ }^{1}$ Prince of Songkla University Faculty of Medicine \\ ${ }^{2}$ Prince of Songkla University
}

January 29, 2022

\begin{abstract}
BACKGROUND: Few studies have examined survival in relapsed childhood acute lymphoblastic leukemia (ALL) in resourcelimited countries. The aims of this study were to evaluate the incidence, prognostic factors, and survival of relapsed childhood ALL in Thailand. METHODS: The medical records of patients with ALL aged $<15$ years in the major tertiary care institution in Southern Thailand between January 2000 and December 2019 were retrospectively reviewed. The Kaplan-Meier method was used to depict the overall survival (OS). RESULTS: A total of 472 patients with ALL were enrolled. The incidence of relapsed ALL was $32.8 \%$. Of the 155 relapsed patients, 131 (84.5\%) and 24 (15.5\%) had B-cell and T-cell phenotypes, respectively. One hundred thirteen $(72.9 \%)$ and $42(27.1 \%)$ patients had early and late relapses, respectively. The most common site of relapse was bone marrow in 102 patients $(65.8 \%)$. One hundred twenty-eight $(82.6 \%)$ and $27(17.4 \%)$ patients received or refused the relapse chemotherapy, respectively. The 5-year OS of all relapsed patients was $11.9 \%$. The 5-year OS among the patients with early relapse was significantly lower than in the patients with late relapse $(5.3 \%$ vs. $29.1 \%$, respectively, p <0.0001). Site and immunophenotype were not associated with survival of relapsed ALL. The patients who refused chemotherapy had a median survival time of 3.1 months. CONCLUSION: The relapse rate was one third of patients with ALL. The 5-year OS was $12 \%$ and patients who refused chemotherapy had a median survival time of 3 months.
\end{abstract}

\section{Relapsedchildhood acute lymphoblastic leukemia: Experience from a single tertiary center in Thailand}

Thirachit Chotsampancharoen ${ }^{1}$, Natsaruth Songthawee ${ }^{1}$, Shevachut Chavananon ${ }^{1}$, Pornpun Sripornsawan ${ }^{1}$, Edward B. McNeil ${ }^{2}$

${ }^{1}$ Division of Hematology and Oncology, Department of Pediatrics, Prince of Songkla University, Hat Yai, Thailand

${ }^{2}$ Epidemiology Unit, Faculty of Medicine, Prince of Songkla University, Hat Yai, Thailand

\section{Correspondence}

Thirachit Chotsampancharoen, Division of Hematology and Oncology, Department of Pediatrics, Faculty of Medicine, Prince of Songkla University, Hat Yai, Songkhla 90110, Thailand. Tel. 66-74-451251, Fax. 66-74-429618, Email: cthirachit@yahoo.com

Word count for abstract $=238$

Word count for main text $=2006$

Number of Tables $=1$ 
Number of Figures $=3$

Short running title: Relapsed childhood acute lymphoblastic leukemia

Keywords: relapsed childhood acute lymphoblastic leukemia, survival outcome, resource-limited countries Abbreviations

\begin{tabular}{ll}
\hline Abbreviation & Full term or phrase \\
\hline ALL & acute lymphoblastic leukemia \\
BFM & Berlin-Frankfurt-Münster \\
CCG & Children's Cancer Group \\
CNS & central nervous system \\
COG & Children's Oncology Group \\
CR1 & first complete remission \\
CR2 & second complete remission \\
CSF & cerebrospinal fluid \\
HSCT & hematopoietic stem cell transplantation \\
IQR & interquartile range \\
MRD & minimal residual disease \\
OS & overall survival \\
POG & Pediatric Oncology Group \\
\hline
\end{tabular}

\begin{abstract}
BACKGROUND: Few studies have examined survival in relapsed childhood acute lymphoblastic leukemia (ALL) in resource-limited countries. The aims of this study were to evaluate the incidence, prognostic factors, and survival of relapsed childhood ALL in Thailand.
\end{abstract}

METHODS: The medical records of patients with ALL aged $<15$ years in the major tertiary care institution in Southern Thailand between January 2000 and December 2019 were retrospectively reviewed. The KaplanMeier method was used to depict the overall survival (OS).

RESULTS: A total of 472 patients with ALL were enrolled. The incidence of relapsed ALL was $32.8 \%$. Of the 155 relapsed patients, $131(84.5 \%)$ and 24 (15.5\%) had B-cell and T-cell phenotypes, respectively. One hundred thirteen $(72.9 \%)$ and $42(27.1 \%)$ patients had early and late relapses, respectively. The most common site of relapse was bone marrow in 102 patients $(65.8 \%)$. One hundred twenty-eight $(82.6 \%)$ and $27(17.4 \%)$ patients received or refused the relapse chemotherapy, respectively. The 5-year OS of all relapsed patients was $11.9 \%$. The 5 -year OS among the patients with early relapse was significantly lower than in the patients with late relapse $(5.3 \%$ vs. $29.1 \%$, respectively, $p<0.0001)$. Site and immunophenotype were not associated with survival of relapsed ALL. The patients who refused chemotherapy had a median survival time of 3.1 months.

CONCLUSION: The relapse rate was one third of patients with ALL. The 5-year OS was $12 \%$ and patients who refused chemotherapy had a median survival time of 3 months.

\title{
INTRODUCTION
}

Recent advances in the treatment of childhood acute lymphoblastic leukemia (ALL) has significantly improved outcomes with long-term survival of greater than $90 \%$ in developed countries. ${ }^{1,2}$ However, the survival in resource-limited countries is significantly lower compared to developed countries with long-term survival of around $70 \% \cdot{ }^{3-7}$ Various studies have reported relapse rates of $10-40 \%$ in patients with childhood ALL, which remains the most common cause of treatment failure, and these patients are more likely to die. ${ }^{3-12}$ Patients with relapsed ALL have inferior outcomes with long-term survival of $58-72 \%$ in developed countries, ${ }^{10,13,14}$ which is higher than the survival in resource-limited countries with long-term survival of 
only $27-42 \%{ }^{6-9,15}$ The factors influencing survival in relapsed ALL include immunophenotype, site and timing of relapse, and treatment. Early relapse, isolated bone marrow relapse, and T-cell phenotype are associated with poor prognosis, ${ }^{11,16}$ while patients who have low minimal residual disease (MRD) after reinduction and who receive hematopoietic stem cell transplantation (HSCT) have improved survival. ${ }^{10,14,15,17}$ Another important prognostic factor found in resource-limited countries which is not so common in developed countries is high rates of chemotherapy refusal. There are few studies which have evaluated survival among patients with relapsed ALL in resource-limited countries, with only one of these evaluating the effect of refusal of chemotherapy on survival. ${ }^{15}$ Thus, studies including patients with relapsed childhood ALL in resource-limited countries are needed to further elucidate the poor outcomes and evaluate the survival among patients with relapsed ALL who refuse chemotherapy. This study aimed to examine the incidence, prognostic factors, and survival of relapsed childhood ALL in Thailand.

\section{MATERIALS AND METHODS}

\section{Patients}

We retrospectively reviewed the medical records of all patients aged under 15 years diagnosed with ALL between January 2000 and December 2019 at Songklanagarind Hospital, the major tertiary health care institution and a university hospital in Southern Thailand.

The clinical characteristics and survival outcomes of patients with relapsed ALL were collected including age, gender, timing of initial diagnosis, immunophenotype, time from initial diagnosis to relapse, site of relapse, treatment for relapse, and survival status at the last follow up.

Diagnosis of newly diagnosed $A L L$

The initial diagnoses of ALL were made based on pathological and immunochemical evaluations of the bone marrow material from bone marrow aspiration and/or biopsy, with the criterion of $>25 \%$ lymphoblasts for the definitive diagnosis. Immunophenotypic classification was divided into two distinct subtypes based on the expression of cell surface markers, B-cell and T-cell.

Chemotherapy protocols for newly diagnosed $A L L$

The chemotherapy protocols used to treat patients with newly diagnosed ALL were divided into 3 periods as follows. Between 2000 and 2005, the patients received the Children's Cancer Group (CCG)-104, CCG-105 and CCG-106 regimens for good, intermediate and poor prognosis, respectively. ${ }^{18,19}$ During the period 20062013, the patients received the CCG-104, CCG-105 and Pediatric Oncology Group (POG) 9006 regimens for standard, high and very high risks, respectively. ${ }^{20,21}$ From 2014-2019, the patients received the Children's Oncology Group (COG)-AALL00P2 regimen for standard risk and the COG-AALL0232 regimen for high and very high risks. ${ }^{1}$ Patients were defined as having achieved a first complete remission (CR1) if they had $<5 \%$ blast cells in the bone marrow and no blast cells in the cerebrospinal fluid (CSF) at the end of the induction phase.

Diagnosis of relapsed $A L L$

The diagnosis of relapse could be made at any time point after achieving CR1. Isolated bone marrow relapse was defined as the presence of $>25 \%$ blast cells in the bone marrow. Isolated central nervous system (CNS) relapse was defined as the presence of [?]5/ $\mu \mathrm{L}$ leukocytes with detectable blast cells in a cytocentrifuged preparation of CSF, or the presence of cranial nerve palsies. An isolated testicular relapse was defined as the presence of unilateral or bilateral testicular enlargement, with biopsy-proven testicular involvement in the absence of bone marrow involvement ( $<5 \%$ blast cells). Combined relapse was defined as the presence of [?] $5 \%$ blast cells in the bone marrow and extramedullary disease relapse. ${ }^{11}$ The timing of relapse was classified according to the COG as early (within 36 months after initial diagnosis) or late bone marrow relapse (after 36 months from initial diagnosis). Isolated extramedullary relapse was defined as early (within 18 months after initial diagnosis) or late relapse (after 18 months from initial diagnosis). ${ }^{22}$

Chemotherapy protocols for relapsed $A L L$ 
The chemotherapy protocols used to treat patients with relapsed ALL were divided into 2 periods as follows. Between 2000 and 2013, relapsed patients received the ALL-REZ Berlin-Frankfurt-Münster (BFM) 87 regimen. ${ }^{23}$ In the period from 2014-2019, they received the UKALL R3 chemotherapy regimen. ${ }^{13}$ Patients were defined as having achieved a second complete remission (CR2) if they had $<5 \%$ blast cells in the bone marrow and no blast cells in the CSF at the end of the re-induction phase.

\section{Statistical analysis}

Descriptive statistics are presented using mean and standard deviation or median and interquartile range (IQR) for continuous variables as appropriate, and frequency with percentage for categorical variables. The Kaplan-Meier method was used to depict the overall survival (OS) from the time of diagnosis of relapse to various time points. The log rank test was used for comparing survival between groups. A $p$-value less than 0.05 was considered significant.

\section{RESULTS}

A total of 501 patients were diagnosed with ALL in our center during the 20-year study period. We excluded 29 patients who had incomplete data, leaving a total of 472 patients for analysis. Of these, $155(32.8 \%)$ had relapsed ALL at a median age of 7.8 years (IQR 5.2-10.8). The percentages of males and females were $60 \%$ and $40 \%$, respectively. Of the 155 relapsed patients, 131 (84.5\%) had the B-cell phenotype and $24(15.5 \%)$ had the T-cell phenotype. Of these, $113(72.9 \%)$ had early relapse with a mean time from initial diagnosis of $1.3 \pm 0.8$ years and $42(27.1 \%)$ had late relapse with a mean time from initial diagnosis of $4.9 \pm 2.1$ years. The most common site of relapse was bone marrow in 102 patients $(65.8 \%)$ followed by combined sites in 28 patients $(18.1 \%)$, CNS in 21 patients (13.5\%) and testis/es in 4 patients $(2.6 \%)$.

Of the 155 relapsed patients, $128(82.6 \%)$ received relapse chemotherapy while 27 (17.4\%) refused chemotherapy and chose alternative medicine. Of the 128 patients who received the relapse chemotherapy, $71(55.5 \%)$ achieved CR2, $44(34.3 \%)$ did not, and 13 (10.2\%) died during re-induction. Of the 128 patients who received the relapse chemotherapy, $47(36.7 \%)$ had a second relapse (Table 1$)$.

\section{Survival outcomes}

Of the 155 relapsed patients, $7(4.5 \%)$ were lost to follow-up. The 3-year and 5-year OS of all relapsed patients were $19.2 \%$ and $11.9 \%$, respectively (Figure 1), with a median survival time of 7.4 months (IQR 5.3-13.1). As shown in Figure 2, the timing of relapse was associated with survival of relapsed ALL. The 5 -year OS among patients with early relapse was significantly lower than in patients with late relapse $(5.3 \%$ vs. $29.1 \%$, respectively, $p<0.0001)$. Site and immunophenotype were not associated with survival of relapsed ALL. The difference in survival between the relapsed B-cell and T-cell ALL groups was not significant ( $12.6 \%$ vs. $8.7 \%$, respectively, $p=0.43)$, nor between isolated bone marrow and other site relapses $(11.3 \%$ vs. $12.8 \%$, respectively, $p=0.29$ ). The survival was significantly different between subgroups of relapsed patients categorized by combined timing of relapse and immunophenotype. The 5-year OS in early B- or T-cell and late B- or T-cell relapses were $4.0 \%, 10.5 \%, 32.3 \%$ and $25 \%$, respectively $(p<0.0001)$.

Among the patients who received the relapse chemotherapy, the median survival times were 5.0 and 1.8 years for the patients who achieved CR2 and who did not, respectively. As shown in Figure 3, the 5-year OS among the patients who achieved CR2 was significantly higher than in the patients who did not (49.4\% vs. $9.1 \%$, respectively, $p<0.0001$ ). Among the patients who did not achieve CR2, all died. Of the 27 patients who refused chemotherapy and chose alternative medicine, the median survival time was 3.1 months.

\section{DISCUSSION}

In our study, the relapse rate of ALL was $33 \%$, which was higher than most studies from developed countries with reported rates ranging from $15 \%$ to $20 \%{ }^{10,11}$ However, the relapse rate in our study was similar to most studies from resource-limited countries with reported rates ranging from $19 \%$ to $40 \%{ }^{3,4,6,7}$ The percentage of relapsed patients who refused chemotherapy in our study was $14 \%$, which was significantly higher than a study from the Nordic countries in which none of the patients refused chemotherapy. ${ }^{10}$ However, the 
percentage of patients refusing chemotherapy in our study was significantly lower than a study from China in which $41 \%$ of the patients refused chemotherapy. ${ }^{15}$

The 5-year OS of patients with relapsed ALL in our study was $12 \%$, which was significantly lower than other studies from developed countries with 5 -year OS of $58 \%$ and $65 \%{ }^{10,14}$ The 5 -year OS of our study was also significantly lower compared to other studies in resource-limited countries with rates of $24 \%$ and $42 \%$. ${ }^{7,15}$ The inferior survival outcome in our study was likely due to a lower rate of CR2, and limited availability of risk-adapted, minimal residual disease (MRD)-directed therapy, HSCT and immunotherapy, which can have a significant influence on survival where available. However, the survival outcomes of patients with relapsed ALL in our study were similar to the study of Tuong et al., ${ }^{9}$ which also had limited access to risk-adapted, MRD-directed therapy, HSCT and immunotherapy. The median survival times of our cohort and the study of Tuong et al. ${ }^{9}$ were 7.4 and 7.5 months, respectively, and the 3 -year OSs were $19 \%$ and $27 \%$, respectively.

Our finding regarding the timing of relapse was concordant with other studies which found that early relapse was an adverse prognostic factor of survival. ${ }^{10,11,15,16}$ Although immunophenotypes have been reported in the literature, the findings have been inconsistent, with other studies reporting that relapsed B-cell ALL was associated with better survival. ${ }^{11,16}$ More recent studies by Oskarsson et al. ${ }^{10}$ and Hu et al. ${ }^{15}$ reported no significant differences in survival between patients with relapsed B-cell and T-cell ALL, as in our study in which we also found that immunophenotype was not associated with survival. Site of relapse has also been reported to be associated with survival in the literature, with several studies reporting that isolated bone marrow relapse was associated with the worst prognosis. ${ }^{10,11,15}$ However, our study did not find any survival differences between isolated bone marrow and other relapse site. We found that patients who did not achieve CR2 after re-induction had very poor survival, which was consistent with other studies. ${ }^{13,15,17} \mathrm{Hu}$ et al. ${ }^{15}$ reported that refusal of chemotherapy was associated with very poor survival with a 5 -year OS of only $5 \%$, a rate similar to our study where refusal of chemotherapy was associated with a 5 -year OS of $9 \%$.

Our study demonstrated that the survival of patients with relapsed ALL was poor in the context of lacking risk-adapted, MRD-directed therapy, HSCT, and immunotherapy. Therefore, we suggest that these treatments would be of benefit for patients with relapsed ALL, particularly those who suffer early relapse and have poor prognoses. MRD-directed therapy, HSCT, and immunotherapy should all be included in the treatment plan. Our study also found poorer survival among patients who refused chemotherapy. Thus, another strategy to improve overall survival among patients with relapsed ALL in resource-limited countries would be devising programs to assist healthcare providers convince these patients to accept this life-lengthening treatment.

Our study had some limitations. First, there is the potential bias inherent in all retrospective studies. Second, our study covered a 20-year period in which advances in diagnosis, chemotherapy regimens and supportive care had been made. Therefore, our data may be considered to be heterogenous in this respect. Third, molecular studies and MRD, which are important for determining ALL risk and treatment, were not done in our study. Finally, since our setting was a major tertiary health care institution and university hospital, which receives mostly high-risk and complicated cases with ALL, there is a possibility of referral bias.

\section{CONCLUSION}

The relapse rate was one third of patients with ALL. The 5-year OS was $12 \%$ and the patients who refused chemotherapy had a median survival time of 3 months.

\section{ACKNOWLEDGMENTS}

We would like to thank Mr. Dave Patterson of the Office of International Affairs, Faculty of Medicine, Prince of Songkla University for his English editing.

\section{CONFLICTS OF INTEREST}

This research did not receive any specific grants from any funding agencies in the public, commercial, or not-for-profit sectors. 


\section{ETHICS STATEMENT}

Ethical approval was obtained from the Ethics Committee of the Faculty of Medicine, Prince of Songkla University, Songkhla, Thailand.

\section{AUTHOR CONTRIBUTIONS}

T.C., N.S., S.C., P.S. and E.M. made substantial contributions to conception and design, acquisition of data, and/or analysis and interpretation of data; T.C. and N.S. were primarily responsible for drafting and revising the manuscript for important intellectual content. All authors provided final approval of the manuscript to be submitted for publication.

\section{DATA AVAILABILITY STATEMENT}

The data that support the findings of this study are available on reasonable request from the corresponding author. The data are not publicly available due to privacy or ethical restrictions.

\section{ORCID}

Thirachit Chotsampancharoen https://orcid.org/0000-0002-0111-6318

Natsaruth Songthawee https://orcid.org/0000-0001-9336-6682

\section{REFERENCES}

1. Hunger SP, Lu X, Devidas M, et al. Improved survival for children and adolescents with acute lymphoblastic leukemia between 1990 and 2005: a report from the children's oncology group. J Clin Oncol 2012;30:16631669.

2. Jeha S, Pei D, Choi J, et al. Improved CNS control of childhood acute lymphoblastic leukemia without cranial irradiation: St Jude Total Therapy Study 16. J Clin Oncol 2019;37:3377-3391.

3. Abdelmabood S, Fouda AE, Boujettif F, Mansour A. Treatment outcomes of children with acute lymphoblastic leukemia in a middle-income developing country: high mortalities, early relapses, and poor survival. J Pediatr (Rio J) 2020;96:108-116.

4. Viana SS, de Lima LM, do Nascimento JB, et al. Secular trends and predictors of mortality in acute lymphoblastic leukemia for children of low socioeconomic level in Northeast Brazil. Leuk Res 2015;39:10601065.

5. Al-Hadad SA, Al-Jadiry MF, Ghali HH, et al. Treatment of childhood acute lymphoblastic leukemia in Iraq: a 17-year experience from a single center. Leuk Lymphoma 2021;62:3430-3439.

6. Jaime-Pérez JC, López-Razo ON, García-Arellano G, et al. Results of treating childhood acute lymphoblastic leukemia in a low-middle income country: 10 year experience in Northeast Mexico. Arch Med Res 2016;47:668-676.

7. Jaime-Pérez JC, Pinzón-Uresti MA, Jiménez-Castillo RA, Colunga-Pedraza JE, González-Llano Ó, Gómez-Almaguer D. Relapse of childhood acute lymphoblastic leukemia and outcomes at a reference center in Latin America: organomegaly at diagnosis is a significant clinical predictor. Hematology 2018;23:1-9.

8. Zapata-Tarrés M, Balandrán JC, Rivera-Luna R, Pelayo R. Childhood acute leukemias in developing nations: successes and challenges. Curr Oncol Rep 2021;23:56.

9. Tuong PN, Kiem Hao T, Kim Hoa NT. Relapsed childhood acute lymphoblastic leukemia: a singleinstitution experience. Cureus 2020;12:e9238.

10. Oskarsson T, Söderhäll S, Arvidson J, et al. Relapsed childhood acute lymphoblastic leukemia in the Nordic countries: prognostic factors, treatment and outcome. Haematologica 2016;101:68-76. 
11. Locatelli F, Schrappe M, Bernardo ME, Rutella S. How I treat relapsed childhood acute lymphoblastic leukemia. Blood 2012;120:2807-2816.

12. Olbara G, van der Wijk T, Njuguna F, et al. Childhood acute lymphoblastic leukemia treatment in an academic hospital in Kenya: treatment outcomes and health-care providers' perspectives. Pediatr Blood Cancer 2021;68:e29366.

13. Parker C, Waters R, Leighton C, et al. Effect of mitoxantrone on outcome of children with first relapse of acute lymphoblastic leukaemia (ALL R3): an open-label randomised trial. Lancet 2010;376:2009-2017.

14. Lew G, Chen Y, Lu X, et al. Outcomes after late bone marrow and very early central nervous system relapse of childhood B-acute lymphoblastic leukemia: a report from the Children's Oncology Group phase III study AALL0433. Haematologica 2021;106:46-55.

15. Hu Q, Hu W, Chen X, et al. Relapsed childhood acute lymphoblastic leukemia: current situation in China; a multicenter observational study. Pediatr Hematol Oncol J 2018;3:59-63.

16. Hunger SP, Raetz EA. How I treat relapsed acute lymphoblastic leukemia in the pediatric population. Blood 2020;136:1803-1812.

17. Eckert C, von Stackelberg A, Seeger K, et al. Minimal residual disease after induction is the strongest predictor of prognosis in intermediate risk relapsed acute lymphoblastic leukaemia - long-term results of trial ALL-REZ BFM P95/96. Eur J Cancer 2013;49:1346-1355.

18. Gaynon PS, Steinherz PG, Bleyer WA, et al. Intensive therapy for children with acute lymphoblastic leukaemia and unfavourable presenting features. Early conclusions of study CCG-106 by the Children's Cancer Study Group. Lancet 1988;2:921-924.

19. Gaynon PS, Bleyer WA, Steinherz PG, et al. Modified BFM therapy for children with previously untreated acute lymphoblastic leukemia and unfavorable prognostic features. Report of Children's Cancer Study Group Study CCG-193P. Am J Pediatr Hematol Oncol 1988;10:42-50.

20. Tubergen DG, Gilchrist GS, O'Brien RT, et al. Improved outcome with delayed intensification for children with acute lymphoblastic leukemia and intermediate presenting features: a Children's Cancer Group phase III trial. J Clin Oncol 1993;11:527-537.

21. Lauer S, Shuster J, Mahoney D, et al. A comparison of early intensive methotrexate/ mercaptopurine with early intensive alternating combination chemotherapy for high-risk B-precursor acute lymphoblastic leukemia: a Pediatric Oncology Group phase III randomized trial. Leukemia 2001;15:1038-1045.

22. Malempati S, Gaynon PS, Sather H, La MK, Stork LC, Children's Oncology Group. Outcome after relapse among children with standard-risk acute lymphoblastic leukemia: Children's Oncology Group study CCG-1952. J Clin Oncol 2007;25:5800-5807.

23. Bührer C, Hartmann R, Fengler R, et al. Importance of effective central nervous system therapy in isolated bone marrow relapse of childhood acute lymphoblastic leukemia. BFM (Berlin-Frankfurt-Münster) Relapse Study Group. Blood 1994;83:3468-3472.

\section{LEGENDS}

TABLE 1 Characteristics of patients with relapsed ALL

FIGURE 1 Kaplan-Meier survival curve of total patients with relapsed acute lymphoblastic leukemia

FIGURE 2 Kaplan-Meier survival curves stratified by timing of relapse between patients with early and late relapsed acute lymphoblastic leukemia

FIGURE 3 Kaplan-Meier survival curves stratified by status of second complete remission (CR2) between patients who achieved CR2 and who did not 


\section{Hosted file}

Table 1 characteristics of relased ALL.docx available at https://authorea.com/users/457955/ articles/554582-relapsed-childhood-acute-lymphoblastic-leukemia-experience-from-asingle-tertiary-center-in-thailand

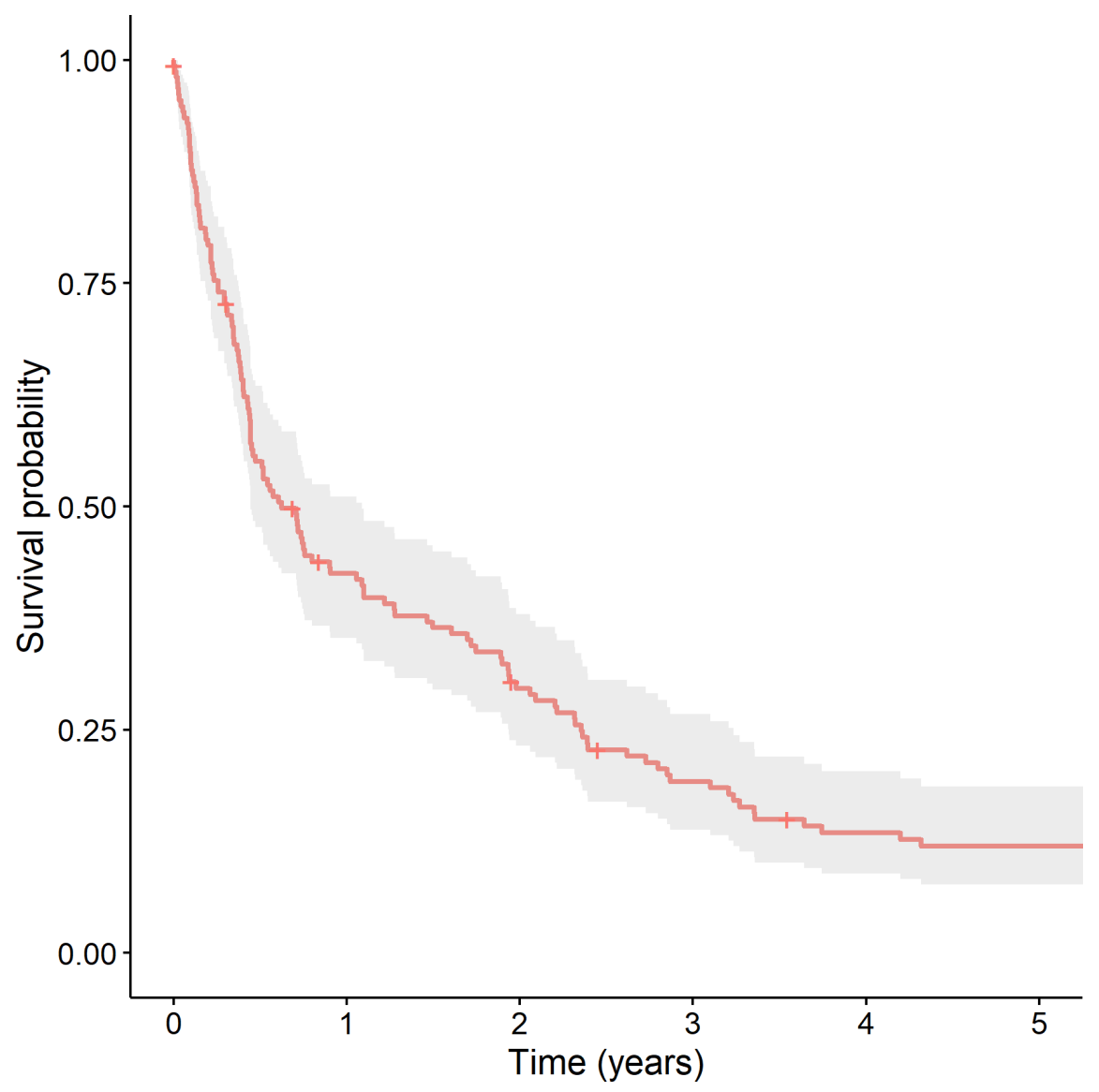




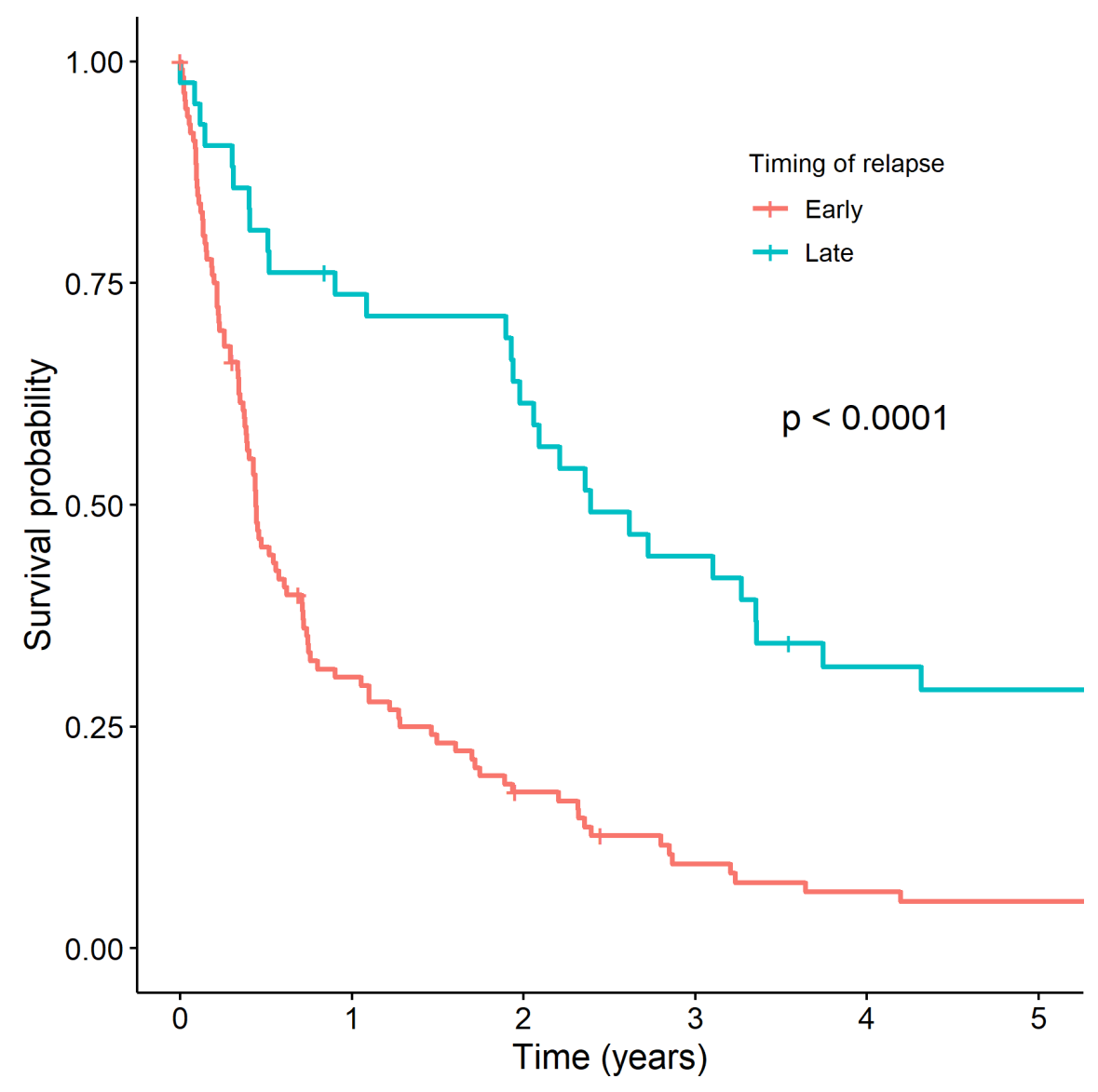




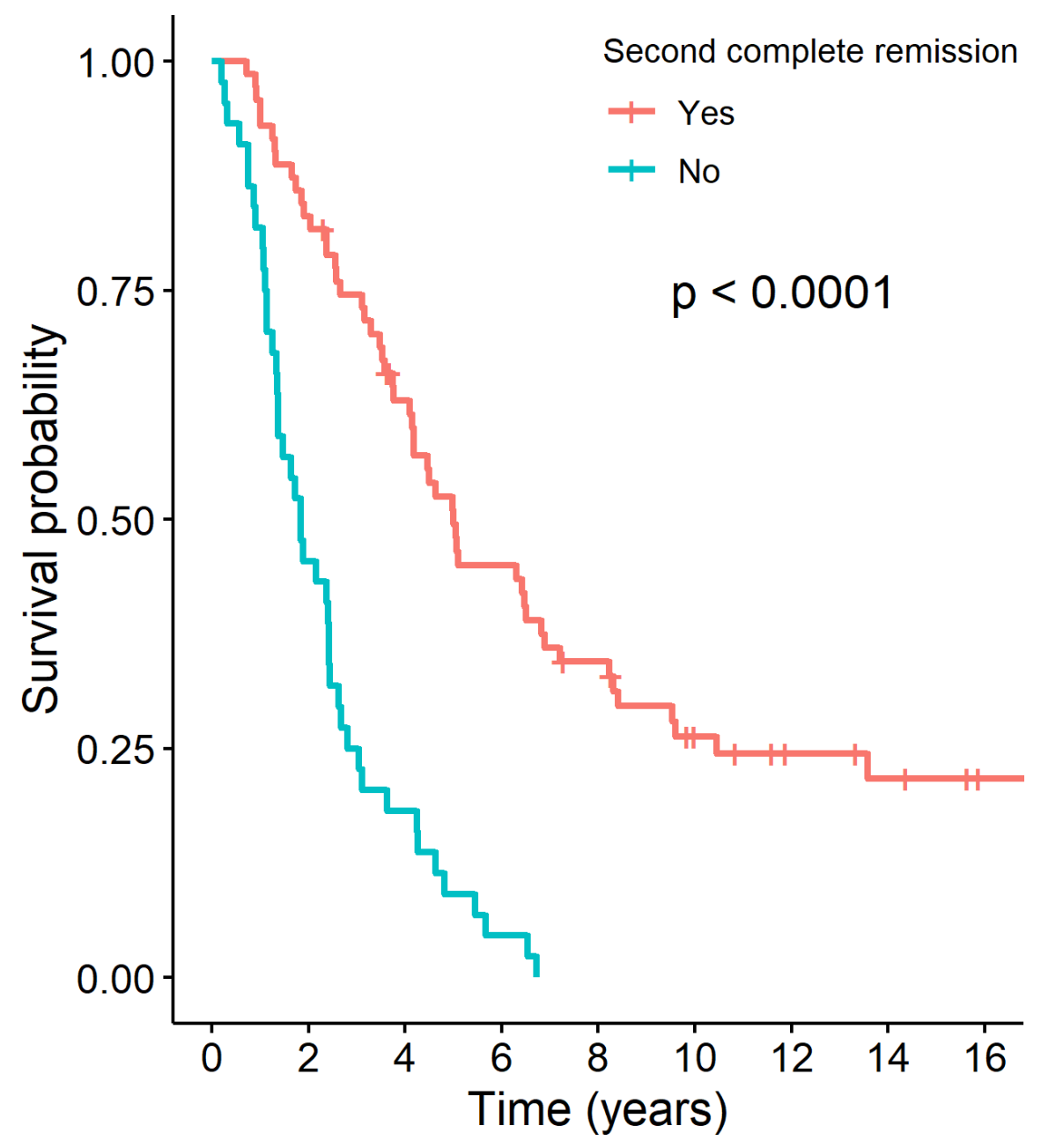

